\title{
Upgrading Physical Layer of Multi-Carrier OGFDM Waveform for Improving Wireless Channel Capacity of 5G Mobile Networks and Beyond ${ }^{\dagger}$
}

\author{
Mohammad R. Kadhum \\ Faculty of Arts, Science \& Technology, University of Northampton, Northampton NN1 5PA, UK; \\ mohammad.kadhum@northampton.ac.uk \\ + This paper is an extended version of my published paper 'New Multi-Carrier Candidate Waveform For the 5G \\ Physical Layer of Wireless Mobile Networks'. In the proceedings of 11th IFIP Wireless Days 2019 conference, \\ Manchester, UK, IEEE, 24-26 April 2019.
}

Received: 29 October 2019; Accepted: 6 January 2020; Published: 10 January 2020

\begin{abstract}
On the brink of sophisticated generations of mobile starting with the fifth-generation (5G) and moving on to the future mobile technologies, the necessity for developing the wireless telecommunications waveform is extremely required. The main reason beyond this is to support the future digital lifestyle that tends principally to maximize wireless channel capacity and number of connected users. In this paper, the upgraded design of the multi-carrier orthogonal generalized frequency division multiplexing (OGFDM) that aims to enlarge the number of mobile subscribers yet sustaining each one with a high transmission capacity is presented, explored, and evaluated. The expanded multi-carrier OGFDM can improve the performance of the future wireless network that targets equally the broad sharing operation (scalability) and elevated transmission rate. From a spectrum perspective, the upgraded OGFDM can manipulate the side effect of the increased number of network subscribers on the transmission bit-rate for each frequency subcarrier. This primarily can be achieved by utilizing the developed OGFDM features, like acceleration ability, filter orthogonality, interference avoidance, subcarrier scalability, and flexible bit loading. Consequently, the introduced OGFDM can supply lower latency, better BW efficiency, higher robustness, wider sharing, and more resilient bit loading than the current waveform. To highlight the main advantages of the proposed OGFDM, the system performance is compared with the initial design of the multicarrier OGFDM side by side with the $5 \mathrm{G}$ waveform generalized frequency division multiplexing (GFDM). The experimented results show that by moving from both the conventional OGFDM and GFDM with $4 \mathrm{GHz}$ to the advanced OGFDM with $6 \mathrm{GHz}$, the gained channel capacity is improved. Because of the efficient use of Hilbert filters and improved rate of sampling acceleration, the upgraded system can gain about $3 \mathrm{~dB}$ and $1.5 \mathrm{~dB}$ increments in relative to the OGFDM and GFDM respectively. This, as a result, can maximize mainly the overall channel capacity of the enhanced OGFDM, which in turn can raise the bit-rate of each user in the mobile network. In addition, by employing the OGFDM with the dual oversampling, the achieved channel capacity in worst transmission condition is increased to around six and twelve times relative to the OGFDM and GFDM with the normal oversampling. Furthermore, applying the promoted OGFDM with the adaptive modulation comes up with maximizing the overall channel capacity up to around $1.66 \mathrm{~dB}$ and $3.32 \mathrm{~dB}$ compared to the initial OGFDM and GFDM respectively. A MATLAB simulation is applied to evaluate the transmission performance in terms of the channel capacity and the bit error rate (BER) in an electrical back-to-back wireless transmission system.
\end{abstract}

Keywords: orthogonal generalized frequency division multiplexing; generalized frequency division multiplexing; Hilbert filter; oversampling factor; adaptive modulation; future mobile waveforms; wireless networks; physical layer; bit error rate 


\section{Introduction}

The upcoming generations of mobile networks tend broadly to keep up the growing requirements of future transmission [1]. This is, therefore, motivating the wireless research community to investigate new techniques for accommodating the key predicated scenarios of modern wireless networks like the bit-pipe communication [2], machine type communication [3], tactile Internet [4], and wireless regional area network [5]. From the physical layer (PHY) perspective, the currently employed orthogonal frequency division multiplexing (OFDM) waveform cannot be able to achieve the future mobile market demands [6]. This mainly results from some significant issues with the OFDM design that makes waveform suffers from the out of band emission, high peak to average power ratio, frequency offset sensitivity, and partially lost bandwidth (BW) [7].

As a result, filtered candidate waveforms with developed features have been introduced recently for the next generations of mobile. For example, the filter bank multi-carrier (FBMC) [8], the universal filter multi-carrier (UFMC) [9], the filtered OFDM (F-OFDM) [10], and the generalized frequency division multiplexing (GFDM) [11]. Consequently, the forthcoming generation of mobile networks can be described as a filtration era. For more clarification, the digital filtration has been applied on different levels of subcarrier allocation. Thus, waveform developers have utilized the filtration either for each orthogonal subcarrier as in the FBMC [12], or for each fixed group of orthogonal subcarriers like in the UFMC [13], or for each flexible group of orthogonal subcarriers as in the F-OFDM [14]. Furthermore, because of the confliction between the employed digital filters and the orthogonal subcarriers, the filtration is applied for each un-orthogonal subcarrier like in the GFDM that has been considered recently for the $5 \mathrm{G}$ mobile networks [15]. However, because of removing the orthogonality with the GFDM waveform, the BW efficiency has been influenced severely.

To address this problem, lately, a single carrier candidate waveform named as orthogonal generalized frequency division multiplexing (OGFDM) is proposed [16]. The presented waveform has obtained the orthogonality for the un-orthogonal subcarriers of a single frequency center $(f c)$ of the GFDM. This, as such, comes up with achieving the orthogonality in the filtration level rather than the subcarriers level. The core idea beyond these advanced filters is the phase change which makes them executed simultaneously. As a result, the developed Hilbert filters can be considered as an emerging solution for the degraded BW efficiency of the GFDM.

From the single carrier transmission perspective, the introduced OGFDM doubled the wireless channel capacity of mobile in comparison with the GFDM [16]. Nevertheless, the single carrier scenario cannot be recommended for the higher wireless channel capacity of future mobile communication $(\mathrm{Gb} / \mathrm{s})$. The main reason beyond this is the single carrier with a high transmission rate can be higher impacted by the inter-symbol interference than the low bit-rate since the maximum expected delay of spread is higher than the specified time for each symbol duration [17]. Thus, the system performance in terms of the channel capacity and bit error rate (BER) can be highly influenced by the utilized way of channel participation. To mitigate such an issue, very recently, the single carrier of the OGFDM has been promoted to the multi-carrier system [18]. The preliminary multi-carrier OGFDM has been launched, as the first stage, with sixteen filtered subcarriers and a sampling frequency is equivalent to $4 \mathrm{GHz}$.

In this paper, an extended version of the multi-carrier OGFDM with a double number of filtered subcarriers (thirty-two) and enlarged size of the $\mathrm{F}_{\mathrm{DAC}}$ equals to $6 \mathrm{GHz}$ is experimentally demonstrated. The developed design of the OGFDM with the multi-carrier system involves five important levels of processing which are known as the acceleration level, filtration level, oversampling level, allocation level, and modulation level.

Regarding the acceleration level, the upgraded design of the OGFDM is extra accelerated to provide a wider BW usage and better transmission rate than the initial OGFDM. Concerning the 
filtration level, the advanced Hilbert filters [19] are applied proficiently on the multi-carrier system ensuring an orthogonal transmission with the upgraded design of the OGFDM. As regards to the oversampling level, the flexible oversampling process [20] is adopted to accommodate any probable interference among the increased filters, that in turns can improve the attained BW efficiency. Relating to the allocation level, a dual number of frequency subcarriers are utilized to extend the scalability of the mobile network. About the modulation level, the adaptive modulation scheme [21] is widely utilized with the progressive multi-carrier OGFDM system to achieve an extra enhancement for the capacity of the transmission channel. Hence, depending on the transmission conditions, the frequency subcarrier with the resilient modulation scheme can be reused in a more efficient way than the fixed modulation system [22].

The performance in terms of the channel capacity and the BER of the promoted OGFDM waveform is fundamentally deliberated in the PHY of an electrical back-to-back wireless transmission system. The rest of the paper is organized as follows: Section 2 discusses the key concepts of the proposed system physically and mathematically. Section 3 evaluates experimentally the system performance utilizing a MATLAB simulation. Section 4 summarizes the outlines of the paper.

\section{System Model of Upgraded Multi-Carrier OGFDM}

Following the successful launch of the multi-carrier OGFDM system [18], in this paper, an extended design of the OGFDM waveform is explored physically and mathematically. The developed system aims to increase the number of subscribers yet keeping each user with a high level of channel capacity. As such, despite the enlarged number of the subcarriers, the introduced system aims to sustain the specified bit-rate for each client by expanding the utilized BW, particularly, after achieving good progress in terms of the BW efficiency of the OGFDM [16,18,20,21].

As is clear in Figure 1, on the transmitter side of the multi-carrier OGFDM system, mainly, at the modulation level, the complex numbers of the frequency domain can be generated via applying different sizes of bit token $(\mathrm{N})$ on an input stream of digital data. By adopting such a hybrid modulation with an enhanced channel state, an extra number of bits can be allocated for each employed subcarrier at the acceptable limit of errors. Consequently, the key downside of the conventional bit loading schemes can be mitigated by alternatively employing an adaptive modulation format.

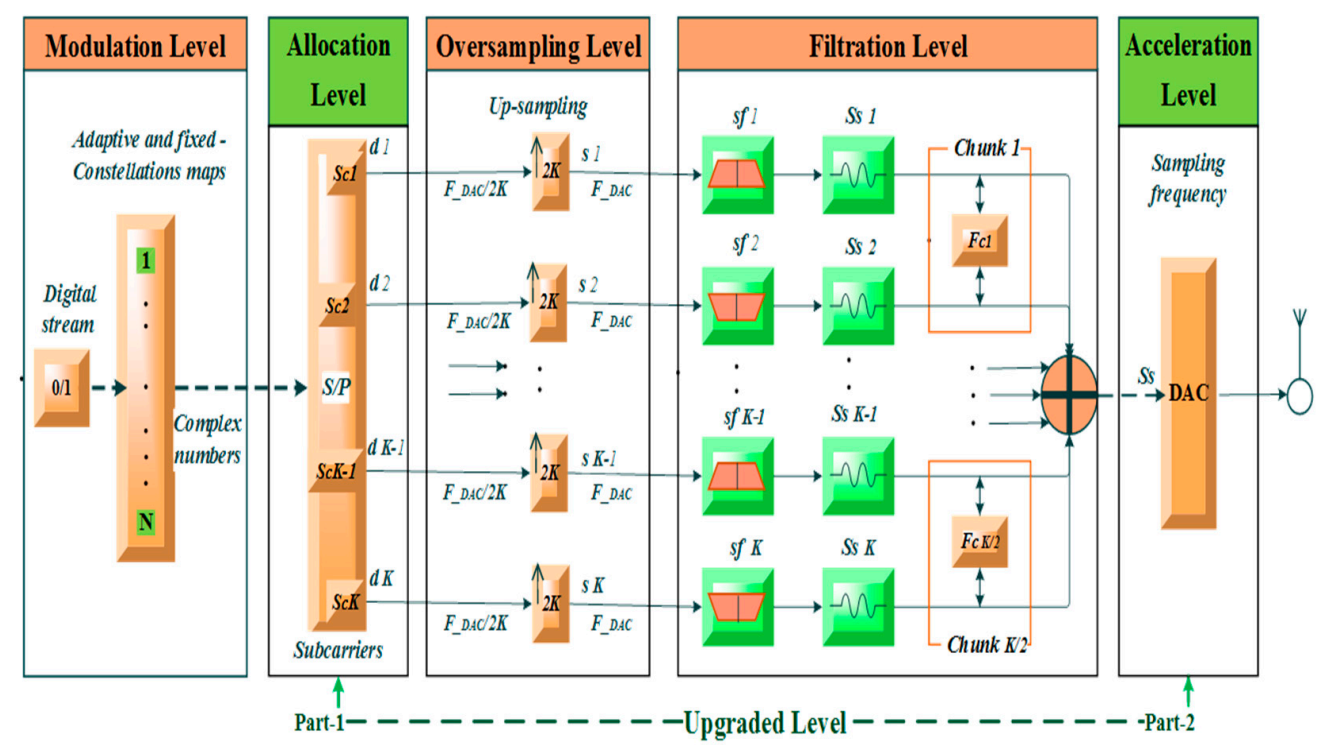

Figure 1. Transmitter design of the orthogonal generalized frequency division multiplexing (OGFDM) including the two key parts of the upgraded level. 
After that, at the allocation level where the first part of upgrading is applied, the obtained complex numbers are distributed among the utilized frequency subcarriers in accordance with their variance ability to carry data. It is worth noting that, the number of used frequency subcarriers is doubled at this level of processing which in turn can increase the number of network subscribers at the upgraded OGFDM.

At the oversampling level, a dual set of the modulated subcarriers is flexibly handled. Thus, the working frequency subcarriers can be up-sampled by a factor of $K$, or $2 K$ according to the system requirements that always tend to achieve a high channel capacity even in worse transmission statuses. The number of assigned copies for each frequency subcarrier is decided by the oversampling stage which represents a significant base for the upcoming stage (filtration level).

Moving to the filtration level where a double assembly of the up-sampled subcarriers is efficiently managed. At this vital stage of processing, every two adjacent subcarriers of frequencies are filtered by the shaping filters of the Hilbert pair and perpendicularly assigned for a similar $f c$. Thus, the cosine and sine parts of the Hilbert filters are employed effectively to orthogonally multiplex the processed subcarriers. The convoluted subcarriers are collected digitally employing a proper electrical adder and input as one sequence of data to the digital-to-analog converter (DAC).

At the acceleration level where the second part of upgrading is applied, the promoted $\mathrm{F}_{\mathrm{DAC}}$ size is utilized to mitigate the impact of system expansion where doubled subcarriers participate in the same resource space. Hence, to avoid sharing limitations, the BW size is amended herein to be compatible with the developed system requirements. Accordingly, the $\mathrm{F}_{\mathrm{DAC}}$ limit should be expanded to keep supplying a high level of bit-rate for each utilized subcarrier. The output of this operation is an analog signal that is ready for transmission by a suitable antenna.

As is seen in Figure 2, on the receiver side of the multi-carrier OGFDM system where the wireless signal is recognized, inverse processes are performed to recover the originally transmitted data. At the acceleration level (the second part of upgrading), the promoted analog-to-digital converter (ADC) is used to convert the analog signal to the digital domain. Then, at the filtration level, the doubled set of the matching filters are employed to extract each intended subcarrier of every elected $f c$. After the de-multiplexing process, the convoluted subcarriers are transferred to the oversampling level where each utilized frequency subcarrier is down-sampled by $K$ or $2 K$ according to the oversampling factor of the transmitter. At the allocation level (the first part of upgrading), the doubled set of the complex numbers that belong to the down-sampled subcarriers are gathered in one stream. At the modulation level, where diffident shapes of modulation are applied, the complex numbers of the frequency subcarriers are converted dynamically into a stream of binary digits. 


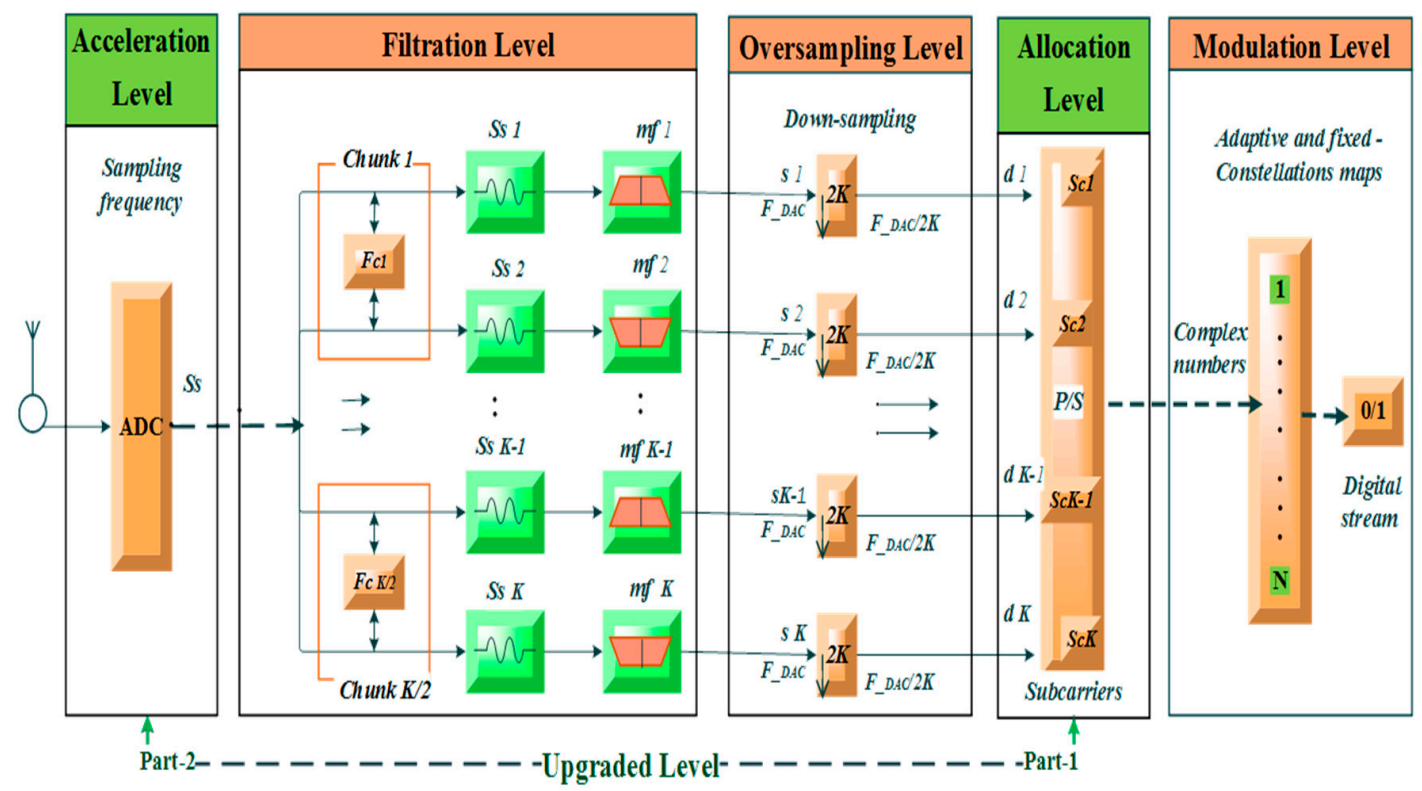

Figure 2. Receiver design of the OGFDM including the two key parts of the upgraded level.

From a mathematical perspective, the multi-carrier system can be expressed as follows:

In the filtration level, the impulse responses of each employed $h$ th pair of the Hilbert filters for shaping filters $(s f)$ are represented as follows [23]:

$$
\begin{aligned}
& s f_{h}{ }^{A}(t)=g(t) \cos \left(2 \pi f c_{h} t\right) \\
& s f_{h}{ }^{B}(t)=g(t) \sin \left(2 \pi f c_{h} t\right)
\end{aligned}
$$

In addition, the impulse responses of matching $(m f)$ are represented as follows [23]:

$$
\begin{aligned}
m f_{h}{ }^{A}(t) & =s f_{h}{ }^{A}(-t) \\
m f_{h}{ }^{B}(t) & =s f_{h}{ }^{B}(-t)
\end{aligned}
$$

where $f c_{h}$ indicates the frequency center of the $h$ th orthogonal pair and, the superscripts $A$ and $B$ refer to the in-phase and out-phase of the applied filter.

Besides, the $g(t)$ signifies the baseband pulse as follows [23]:

$$
g(t)=\frac{\sin [\pi(1-\alpha) \Upsilon]+4 \alpha \curlyvee \cos [\pi(1+\alpha) \Upsilon]}{\pi \curlyvee\left[1-(4 \alpha \Upsilon)^{2}\right]}
$$

where $r=t / \Delta t$, roll-off $(\alpha)$ specifies the filter excess and $\Delta t$ denotes the sampling interval prior to the oversampling process.

Moreover, the output signal of the convolution operation between the shaping and matching filters is expressed as follows [23]:

$$
m f_{j}{ }^{C}(t) \otimes s f_{i}{ }^{D}(t)=\left\{\begin{array}{c}
\delta\left(t-t_{0}\right), \text { if } C=D \text { and } j=i \\
0, \text { if } C \neq D \text { or } j \neq i
\end{array}\right\}
$$

where $t_{0}$ states the probable delay, the subscripts $i$ and $j$ represent the order of the $f c$, and the superscripts $C$ and $D$ indicates either the in-phase or out-phase.

Considering that the expanded frequency sampling is equivalent in both sides (transmitter and receiver), the $f c$ of each filter pair is allocated as follows [24]:

$$
f_{C_{h}}=(2 h-1)(\mathrm{BW} / \mathrm{K})
$$


where, $h$ denotes the position of the Hilbert pair and BW equals to $F_{D A C / A D C} / 2$.

Since every applied $f_{C}$ is optimally selected, the utilized Hilbert filters are accommodated orthogonally and distributed sequentially in the available spectrum.

The specified BW of each employed filter $\left(F_{B W}\right)$ can be expressed as follows [25]:

$$
F_{B W}=S u b_{S} *(1+\alpha)
$$

where $1 \leq \alpha \leq 0$, and the frequency sampling of subcarrier $\left(S u b_{S}\right)$ represents the size of generated copy for each oversampled subcarrier.

In the oversampling level, a flexible oversampling process is applied to decide the required sampling frequency for each frequency subcarrier as follow [25]:

$$
S u b_{S}=F_{D A C} / O V
$$

where $O V$ refers to the oversampling factor that mostly equals to the number of used subcarriers $(K)$.

Besides, this important factor $(\mathrm{OV})$ can be employed to determine the number of generated copies for each utilized subcarrier. Occasionally, the $O V$ factor is doubled $(2 K)$ to give extra support for the employed filters, nevertheless, this kind of manipulation can impact the overall channel capacity according to Shannon theorem as follows [26]:

$$
\text { Capacity }=(B W) * \log 2(1+S N R)
$$

\section{Experimental Work}

In this part, the expanded multi-carrier OGFDM system is experimentally demonstrated to evaluate the performance in terms of the channel capacity and BER. Therefore, a numerical simulation (MATLAB code) is achieved for the developed design of the OGFDM including five levels of processing (acceleration, filtration, oversampling, allocation, and modulation). Furthermore, to highlight the key advantages of the upgraded OGFDM, the performance is compared with 5G waveform (GFDM). Moreover, the enhanced PHY of the OGFDM is investigated in an electrical back-to-back wireless transmission system.

Prior to generating the extended design of the OGFDM, two different design trials are introduced and compared with the initial design case [18]. The main reason behind these tests is to select the optimal PHY that can be favorable to the predicated requirements of future mobile networks. The introduced attempts show that neither an increasing number of subscribers nor supplying higher channel capacity alone can be suitable for the upcoming generations of mobile. Hence, the hybrid solution that combines both the scalability and high transmission rate should be adopted.

To clarify more about the OGFDM expansion and how the developed scenario is nominated, the following experimental cases discuss, from a spectrum perspective, the assigned BW for one selected $f_{c}$ in the examined multi-carrier OGFDM system.

In case 1 (initial state), as is seen in Figure 3, with 16 frequency subcarriers and $\mathrm{F}_{\mathrm{DAC}}$ corresponds to $4 \mathrm{GHz}$, the specified sampling frequency ( $\mathrm{F}_{\mathrm{DATA}}$ ) for one $f c$ is equivalent to $250 \mathrm{MHz}$. As a result, the achieved bit-rate of each frequency subcarrier equals to $875 \mathrm{Mb} / \mathrm{s}$ with aggregated channel capacity equals to $14 \mathrm{~Gb} / \mathrm{s}$. 


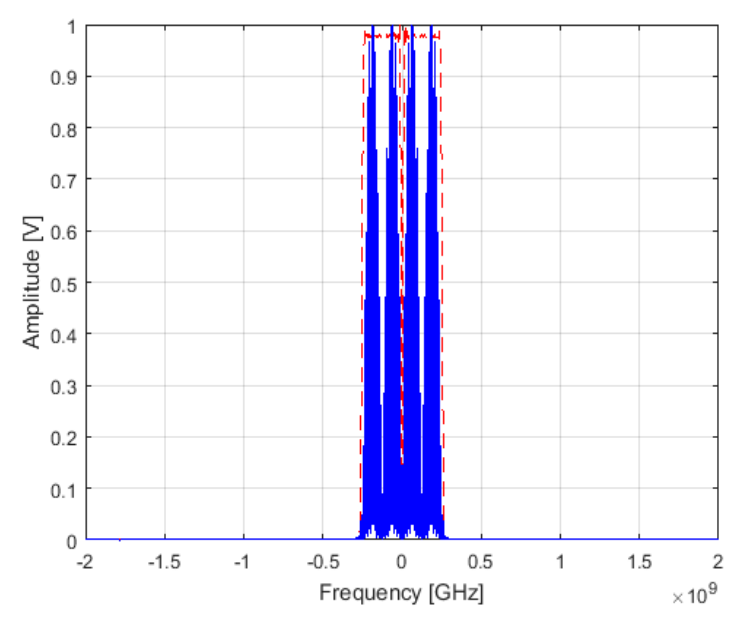

Figure 3. Case 1 (initial): $\mathrm{F}_{\mathrm{DAC}}=4 \mathrm{GHz}, K=16$.

In case 2 (subcarriers number increment), the test proposes to increase the number of frequency subcarriers yet leaving the sampling frequency without a change in comparison with case 1 . As is clear in Figure 4, with a steady level of sampling frequency $\left(\mathrm{F}_{\mathrm{DAC}}=4 \mathrm{GHz}\right)$ and a dual number of subcarriers $(K=32)$, the assigned sampling frequency for one $f c$ is affected badly $\left(\mathrm{F}_{\mathrm{DATA}}=125 \mathrm{MHz}\right)$. Thus, the subcarrier BW is reduced to half of its initial case because of doubling the applied subcarriers without expanding the speed of $\mathrm{F}_{\mathrm{DAC}}$. Despite the overall channel capacity is still compatible with case1, the bit-rate of each applied subcarrier is descended to $437.5 \mathrm{Mb} / \mathrm{s}$ ( $50 \%$ decrement).

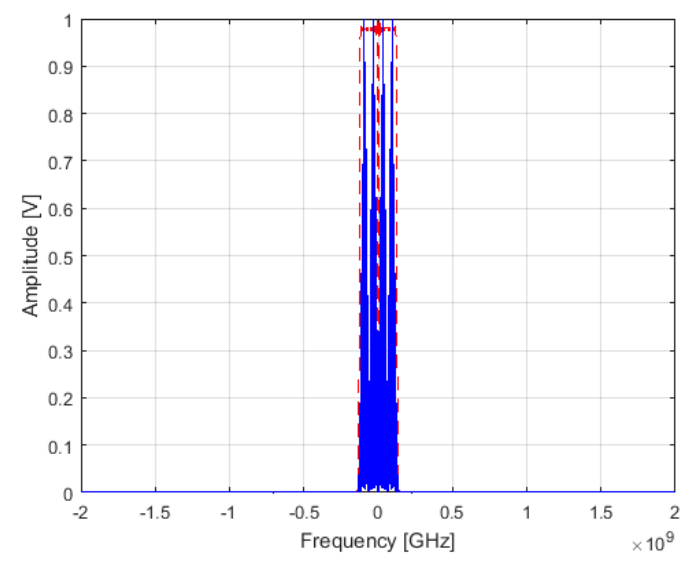

Figure 4. Case 2: $\mathrm{F}_{\mathrm{DAC}}=4 \mathrm{GHz}, K=32$.

In case 3 (sampling frequency and number of subcarriers increment), in comparison with case 1 , the test introduces a suitable enhancement for both the sampling frequency and the number of frequency subcarriers. As is obvious in Figure 5, an advanced case of transmission is gotten herein by expanding the utilized sampling frequency from 4 to $6 \mathrm{GHz}$ and updating the applied number of subcarriers into 32 (double). Worth noting that, the $\mathrm{F}_{\mathrm{DAC}}$ of this case can be further enlarged, but since the multi-carrier OGFDM system tends currently to work at the $6 \mathrm{GHz}$ band radio frequency, the employed $\mathrm{F}_{\mathrm{DAC}}$ is upgraded by only $50 \%$ of the initial case. In comparison to the second case, a better sampling frequency $(187.5 \mathrm{MHz})$ is obtained herein which in turn can increase the bit rate of transmission for each utilized subcarrier to $656.25 \mathrm{Mb} / \mathrm{s}$ side by side with escalating the overall channel capacity to $21 \mathrm{~Gb} / \mathrm{s}$. Since the future applications of mobile tend to increase the number of subscribers yet keeping the bit-rate of each one at a high level, this case that combines both the improved bit-rate and the enlarged number of subcarriers is highly recommended for the expanded design of the multi-carrier OGFDM waveform. 


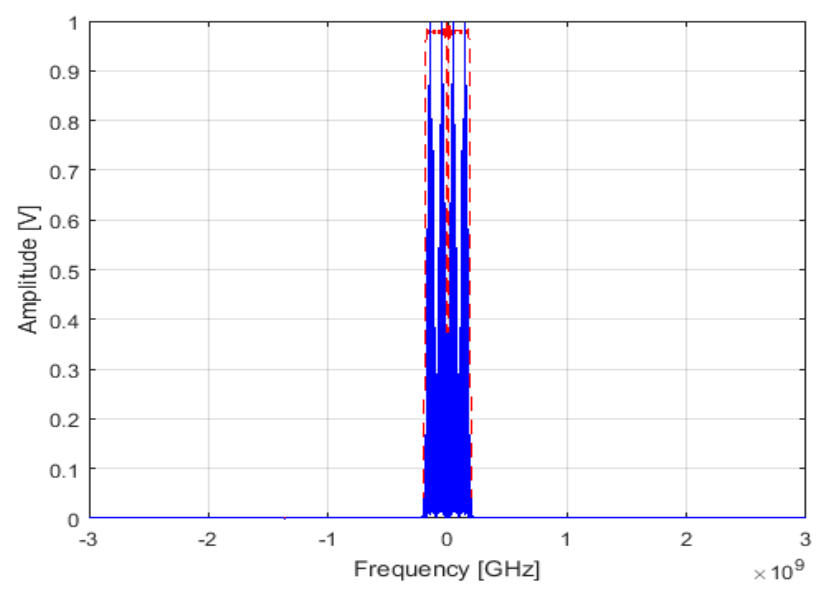

Figure 5. Case 3: $\mathrm{F}_{\mathrm{DAC}}=6 \mathrm{GHz}, \mathrm{K}=32$.

The following Table 1 can summarize the introduced cases for the OGFDM expansion including the initial case.

After selecting the required scenario (case 3) for the developed OGFDM design, the main system parameters are updated accordingly. The impact of the upgraded PHY on the system performance (channel capacity and BER) is explored for the five levels of manipulation. Thus, such important parameters like the number of subcarriers and sampling frequency size can directly influence the acceleration, filtration, oversampling, allocation, and modulation levels of manipulation.

Table 1. Comparison of expansion trials for multi-carrier OGFDM system.

\begin{tabular}{ccccccc}
\hline & F $_{\text {DAC }}(\mathbf{G H z})$ & $\mathbf{N}_{\mathbf{S c}}$ & F $_{\text {DATA }}(\mathbf{M H z})$ & fc $(\mathbf{M H z})$ & Sc-Cap $\mathbf{( M b} / \mathbf{s})$ & T-Cap (Gb/s) \\
\hline Case 1 & 4 & 16 & 250 & 125 & 875 & 14 \\
Case 2 & 4 & 32 & 125 & 62.5 & 437.5 & 14 \\
Case 3 & 6 & 32 & 187.5 & 93.75 & 656.25 & 21 \\
\hline
\end{tabular}

Regarding the acceleration and filtration levels, the developed multi-carrier OGFDM with $6 \mathrm{GHz}$ is mainly compared with the $5 \mathrm{G}$ waveform (GFDM) exploring the impact of Hilbert filters on the transceiver process. In addition, despite that the expanded sampling frequency is set to $6 \mathrm{GHz}$, the effect of extending the initial case of the $\mathrm{F}_{\mathrm{DAC}}$ from 4 to $6 \mathrm{GHz}$ is also considered for the overall performance. Thus, investigating how the change in sampling frequency can impact the bit-rate of transmission in cooperating with the orthogonal filters that play a big role in improving the BW efficiency and then supporting the channel capacity of the upgraded system.

As is seen in Figure 6, in this experiment, a stable $2 \mathrm{~dB}$ gain is obtained between the aggregated channel capacity of the OGFDM and the GFDM because of the orthogonality impact of the utilized Hilbert filters on the OGFDM waveform. Thus, a higher channel capacity (double) is achieved with the orthogonal OGFDM than the non-orthogonal GFDM with that root-raised-cosine (RRC) for an equivalent level of the sampling frequency. In addition, because of expanding the applied $\mathrm{F}_{\mathrm{DAC}}, 3 \mathrm{~dB}$ improvement can be achieved between the OGFDM and the GFDM. The main reason beyond this increment is the combined influence of both the advanced Hilbert filters and the improved sampling frequency. As such, the high channel capacity can be acquired by either improving the BW efficiency (Hilbert pair) or by extra extending the used sampling frequency. 


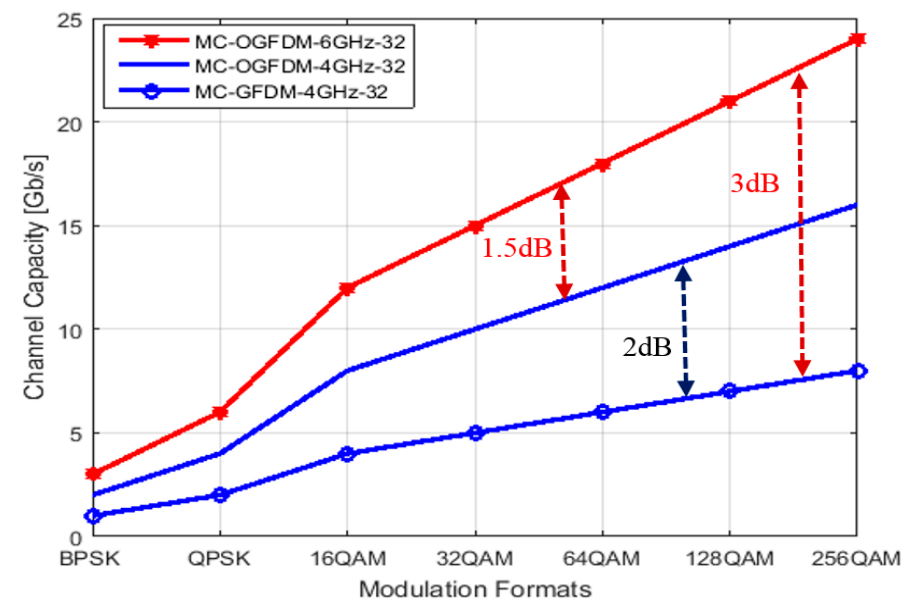

Figure 6. Channel capacity of the multi-carrier OGFDM vs. generalized frequency division multiplexing $(\mathrm{GFDM})$ with $\mathrm{F}_{\mathrm{DAC}}=4$ and $6 \mathrm{GHz}$.

Regarding the oversampling level, with the normal oversampling (NOV), where the oversampling factor $(O V)$ equals to the number of the subcarriers $(K)$, the interference between filtered subcarriers is decided according to the $\alpha$ value. To mitigate such an issue, a dual oversampling (DOV) is adopted where the number of produced copies for each utilized subcarrier can be doubled $(O V=2 K)$ for the same utilized centers of frequencies. Therefore, the negative effect of the $\alpha$ can be accommodated counting on the offered band intervals, which in turn, can remove any possible intra-channel interference.

The upgraded OGFDM is compared with the initial OGFDM and GFDM considering the impact of the hybrid treatment (NOV and DOV) on the system performance (channel capacity and BER) at $6 \mathrm{GHz}$ sampling frequency.

As is shown in Figure 7, the experimental work indicates that by utilizing the DOV with the upgraded OGFDM at worst transmission states $(0.5 \leq \alpha \leq 1)$, the channel capacity is extremely improved. Thus, $6 \mathrm{~dB}$ and $12 \mathrm{~dB}$ gains are obtained for the channel capacity of the extended OGFDM with the DOV higher than the conventional OGFDM and GFDM with the NOV.

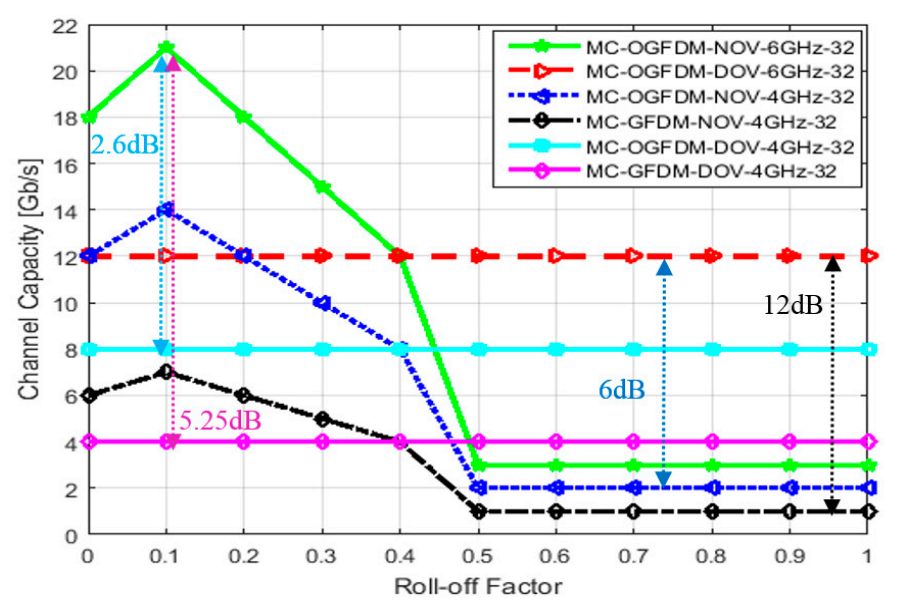

Figure 7. Channel capacity of the multi-carrier OGFDM vs. GFDM with typical and dual oversampling and $\mathrm{F}_{\mathrm{DAC}}=4$ and $6 \mathrm{GHz}$.

Nevertheless, at a good transmission condition $(0 \leq \alpha \leq 0.4)$, the NOV mode can supply a better transmission rate than the DOV. As such, by employing the NOV with the developed OGFDM at the optimum value of the rolling $(\alpha=0.1)$, around $2.6 \mathrm{~dB}$ and $5.25 \mathrm{~dB}$ gains are recorded in comparison with the initial OGFDM and GFDM at DOV mode. As a result, for a robust transmission scenario of the 
OGFDM, a combination of both the NOV and DOV is utilized, where the NOV is highly advised to the good transmission cases while the DOV is strongly recommended for the bad transmission conditions.

Regarding the allocation level, as is seen in Figure 8, three vital areas, that are known herein as low boost (LB), medium boost (MB), and high boost (HB), are elected between the 128 QAM and 256 QAM with minimum SNR edges equal to $23 \mathrm{~dB}$ and $26 \mathrm{~dB}$ correspondingly.

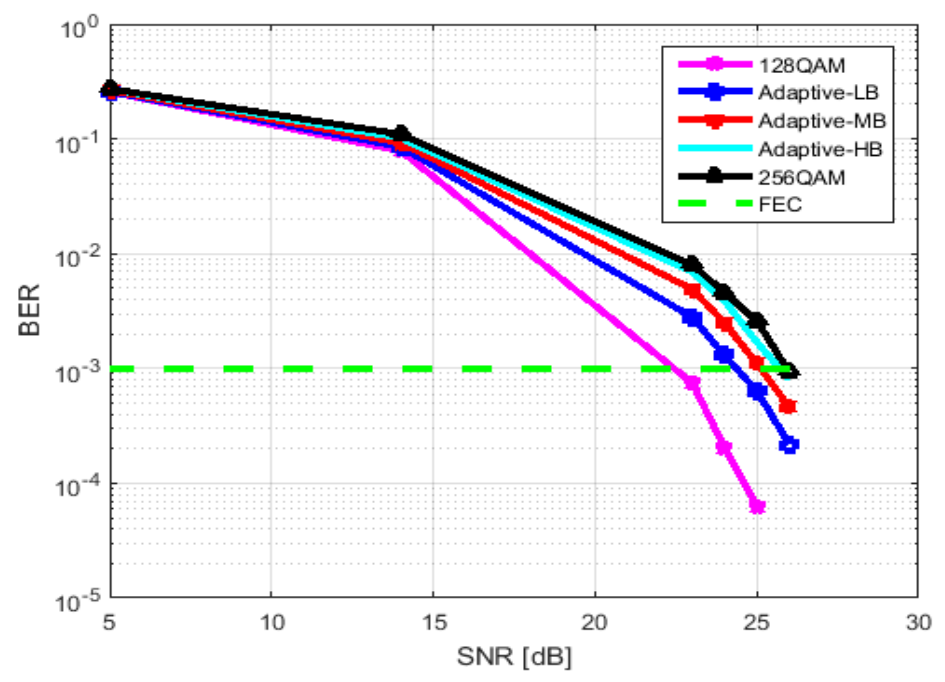

Figure 8. Three significant adaptive areas for the OGFDM, low boost (LB), medium boost (MB), and high boost (HB) between two sequential fixed modulation schemes (128 and 256 QAM).

The first case (LB) is calculated for a $25 \%$-bit-rate improvement with the SNR threshold equals to $24.4 \mathrm{~dB}$ where different possible arrangements of the bit loading map can be shown for this ratio of the enhancement. Worth noting that, compared with the initial OGFDM and GFDM, the number of improved subcarriers that can carry extra bit is doubled and quadrupled respectively. The core idea beyond the LB case is that a quarter of the subcarriers is improved gradually by loading 8 bits rather than 7, which in turns, results in a minor channel capacity increase.

In the second case (MB), the threshold of the required SNR is improved to $25.1 \mathrm{~dB}$. Consequently, based on the amended channel conditions, half of the frequency subcarriers can carry an extra number of bits. This, as a result, develops the transmission capacity of the channel by about $50 \%$ of the total increment that is possibly achieved between the selected modulations. It is worth pointing that in comparison with the previous multi-carrier systems (OGFDM and GFDM), the number of subcarriers with extra bit ability is enhanced for the developed system of the OGFDM.

In the third case (HB), the promoted threshold of the SNR can come up with $75 \%$-bit-rate development in comparison with the previously stated modes (MB, LB). Therefore, at the HB where the recorded SNR equals to $25.1 \mathrm{~dB}$, the number of frequency subcarriers with a further bit is enhanced to 24 improved cases with the developed OGFDM in comparison with only 12 and 6 amended subcarriers with the initial OGFDM and GFDM respectively.

Concerning with the modulation levels, an extra BW efficiency is gained herein by applying the adaptive modulation format on the updated system. Besides, the system performance (channel capacity and BER) of the promoted OGFDM is compared with the conventional OGFDM and GFDM under the fixed and adaptive modulation schemes.

As is shown in Figure 9, the findings of the experiment declare that the upgraded OGFDM with adaptive modulation can extra improve the transmission bit-rate compared to the OGFDM and GFDM with the fixed modulation format. Hence, in the LB case, the developed OGFDM can achieve about $1.55 \mathrm{~dB}$ and $3 \mathrm{~dB}$ gains in comparison with the initial OGFDM and GFDM respectively. Worth noting that, these gained ratios can be maximized up to around $1.66 \mathrm{~dB}$ and $3.32 \mathrm{~dB}$ by moving to the HB. Such a notable improvement is essentially acquired because of the powerful use of the adaptive bit loading 
system, side by side with improving the sampling rate of the OGFDM waveform. The improved channel capacity can play a big role in supplying a good transmission bit rate for each subscriber in the wireless network.

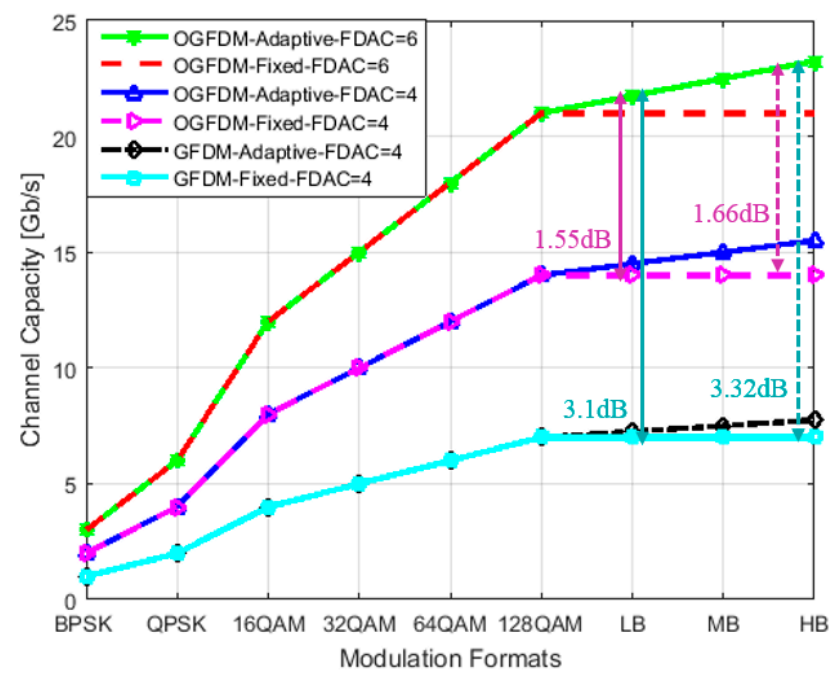

Figure 9. Channel capacity of the multi-carrier OGFDM vs. GFDM with adaptive modulations and $\mathrm{F}_{\mathrm{DAC}}=4$ and $6 \mathrm{GHz}$.

The key conditions of this experimented system are listed in Table 2.

Table 2. System parameters of multi-carrier OGFDM.

\begin{tabular}{cc}
\hline Parameter & Value \\
\hline No. of frequency centers & 16 \\
FDAC/ADC & $6 \mathrm{GHz}$ \\
SNR & $23-26 \mathrm{~dB}$ \\
Roll-off & $0-1$ \\
System mode & Multi-carrier \\
Number of subcarriers & 32 \\
OGFDM symbols & 2000 \\
Filter length & 32 \\
Modulation format & Fixed \& Adaptive \\
Oversampling & Normal (32) \& Dual (64) \\
Filter type & RRC \& Hilbert filters \\
\hline
\end{tabular}

\section{Conclusions}

In this paper, extended version of the multi-carrier OGFDM system with 32 frequency subcarriers and sampling frequency equals to $6 \mathrm{GHz}$ is proposed, examined, and evaluated. The developed scheme can further support a wider number of mobile network users and a higher overall channel capacity than the initial multi-carrier OGFDM. Experimentally speaking, the upgraded OGFDM has five levels of manipulation (acceleration, filtration, oversampling, allocation, and modulation) that directly impacts the performance of the transmission system. Because of the close bonding between the acceleration and filtration levels, the experiment shows $1.5 \mathrm{~dB}$ and $3 \mathrm{~dB}$ improvements via moving from the preliminary OGFDM and GFDM to the developed OGFDM. In the oversampling level, mainly at the bad transmission conditions, the channel capacity of the OGFDM with the proposed dual sampling (DOV) far outweigh the transmission capacity of the OGFDM and GFDM at normal sampling (NOV) by around $6 \mathrm{~dB}$ and $12 \mathrm{~dB}$ respectively. However, at good transmission cases, the findings indicate that by using the NOV with the promoted OGFDM, the recorded bit-rate can be better than the transmission rate of the OGFDM and GFDM with the DOV by about $2.6 \mathrm{~dB}$ and $5.25 \mathrm{~dB}$ correspondingly. As a 
result, the hybrid solution (normal and dual) is recommended with the advanced OGFDM to extremely support the high channel capacity at good and bad transmission conditions. In the allocation and the modulation levels, the results declare that the improved OGFDM can achieve around $1.66 \mathrm{~dB}$ and $3.32 \mathrm{~dB}$ gains in comparison with the initial OGFDM and GFDM. By improving the overall channel capacity of the upgraded OGFDM, a good transmission bit rate can be supplied for each subscriber in the mobile network.

Funding: This research was funded by the Ministry of Higher Education and Scientific Research, Republic of Iraq, grant number 2633 and the APC was funded by the University of Northampton, UK.

Conflicts of Interest: The author declares no conflict of interest.

\section{References}

1. Haboobi, H.; Kadhum, M.R. Impact Study and Evaluation of Higher Modulation Schemes on Physical Layer of Upcoming Wireless Mobile Networks. Int. J. Adv. Comput. Sci. Appl. 2019, 10, 5. [CrossRef]

2. Wan, L.; Guo, Z.; Wu, Y.; Bi, W.; Yuan, W.; Elkashlan, M.; Hanzo, L. 4G/5G Spectrum Sharing. IEEE Veh. Technol. Mag. 2018, 13, 28-39. [CrossRef]

3. Walia, J.S. Future Scenarios and Value Network Configurations for Industrial 5G. In Proceedings of the 2017 8th International Conference on the Network of the Future (NOF), London, UK, 22-24 November 2017; pp. 79-84.

4. Simsek, M.; Aijaz, A.; Dohler, M.; Sachs, J.; Fettweis, G. 5G-Enabled Tactile Internet. IEEE J. Sel. Areas Commun. 2016, 34, 460-473. [CrossRef]

5. Han, B.I.N. A Comprehensive Survey of RAN Architectures Toward 5G Mobile Communication System. IEEE Access 2019, 7, 70371-70421.

6. Yli-Kaakinen, J.; Levanen, T.; Valkonen, S.; Pajukoski, K.; Pirskanen, J.; Renfors, M.; Valkama, M. Efficient fast-convolution-based waveform processing for 5G physical layer. IEEE J. Sel. Areas Commun. 2017, 35, 1309-1326. [CrossRef]

7. Balint, C. OFDM-Based Multi-Carrier Waveforms Performances in 5G. In Proceedings of the 2018 International Symposium on Electronics and Telecommunications, Timisoara, Romania, 8-9 November 2018; pp. 1-4.

8. Gaohui, L. Research on a Modulation Recognition Method for the FBMC-OQAM Signals in 5G Mobile Communication System. In Proceedings of the 2018 13th IEEE Conference on Industrial Electronics and Applications, Wuhan, China, 31 May-2 June 2018; pp. 2544-2547.

9. Saad, M.; Al-ghouwayel, A.; Hijazi, H. UFMC Transceiver Complexity Reduction. In Proceedings of the 2018 25th International Conference on Telecommunications, St. Malo, France, 26-28 June 2018; pp. 295-301.

10. Wu, D.; Zhang, X.; Qiu, J.; Gu, L.; Saito, Y.; Benjebbour, A.; Kishiyama, Y. A field trial of f-OFDM toward 5G. In Proceedings of the 2016 IEEE Globecom Workshps (GC Wkshps), Washington, DC, USA, 4-8 December 2016.

11. Towliat, M.; Mohammad, S.; Asgari, J. GFDM Interference Mitigation without Noise Enhancement. IEEE Commun. Lett. 2018, 22, 1042-1045. [CrossRef]

12. Yunzheng, T.; Long, L.; Shang, L.; Zhi, Z. A Survey: Several Technologies of Non-Orthogonal Transmission for 5G. China Commun. 2015, 12, 1-15.

13. Bochechka, G.; Tikhvinskiy, V.; Vorozhishchev, I.; Aitmagambetov, A.; Nurgozhin, B. Comparative analysis of UFMC technology in 5G networks. In Proceedings of the 2017 International Siberian Conference on Control and Communications, Astana, Kazakhstan, 29-30 June 2017; pp. 1-6.

14. Hazareena, A. A survey: On the waveforms for 5G. In Proceedings of the 2018 Second International Conference on Electronics, Communication and Aerospace Technology, Coimbatore, India, 29-31 March 2018; no. Iceca. pp. 64-67.

15. Hilario-tacuri, A.; Fortes, J.M.P.; Sampaio-neto, R. Analytical spectral evaluation of GFDM systems over non-linear channels with memory. In Proceedings of the 2018 IEEE 10th Latin-American Conference on Communications (LATINCOM), Guadalajara, Mexico, 14-16 November 2018; pp. 1-5.

16. Kadhum, M.R.; Kanakis, T.; Al-sherbaz, A.; Crockett, R. Digital Chunk Processing with Orthogonal GFDM Doubles Wireless Channel Capacity. In Advances in Intelligent Systems and Computing, Proceedings of Computing Conference 2018, London, UK, 10-12 July 2018; Springer: Cham, Switzerland, 2018. 
17. Zhu, L.; Zhang, J.; Pei, Y.; Ge, N.; Lu, J. On Maximum Achievable Information Rates of Single-Carrier and Multi-Carrier Systems over the Ultra-Wideband Channels. In Proceedings of the 2010 IEEE International Conference on Ultra-Wideband, Nanjing, China, 20-23 September 2010; Volume 2, pp. 1-4.

18. Kadhum, M.R. New Multi-Carrier Candidate Waveform for the 5G Physical Layer of Wireless Mobile Networks. In Proceedings of the IFIP Wireless Days 2019, IEEE Conference, Manchester, UK, 24-26 April 2019.

19. Smirnov, P.N.; Kharitonov, S.A.; Preobrajensky, E.B. Synthesis Methods of Hilbert Filters for Control Systems of Static Converters. In Proceedings of the 2015 16th International Conference of Young Specialists on Micro/Nanotechnologies Electron Devices, Erlagol, Russia, 29 June-3 July 2015; pp. 486-489.

20. Kadhum, M.R.; Kanakis, T.; Crockett, R. Intra Channel Interference Avoidance with the OGFDM Boosts Channel Capacity of Future Wireless Mobile Communication. In Advances in Intelligent Systems and Computing, Proceedings of the Computing Conference 2019, London, UK, 16-17 July 2019; Springer: Berlin/Heidelberg, Germany, 2019.

21. Kadhum, M.R.; Kanakis, T.; Crockett, R. Dynamic Bit Loading with the OGFDM Waveform Maximises Bit-Rate of Future Mobile Communications. In Advances in Intelligent Systems and Computing, Proceedings of Computing Conference 2019, London, UK, 16-17 July 2019; Springer: Berlin/Heidelberg, Germany, 2019.

22. Haboobi, H.; Kadhum, M.R. Utilise Higher Modulation Formats with Heterogeneous Mobile Networks Increases Wireless Channel Transmission. In Advances in Intelligent Systems and Computing, Proceedings of Computing Conference 2019, London, UK, 16-17 July 2019; Springer: Berlin/Heidelberg, Germany, 2019.

23. Tao, L.; Wang, Y.; Gao, Y.; Lau, A.P.T.; Chi, N.; Lu, C. Experimental demonstration of 10 Gb/s multi-level carrier-less amplitude and phase modulation for short range optical communication systems. Opt. Express 2013, 21, 6459. [CrossRef] [PubMed]

24. Jin, W.; Zhang, C.; Duan, X.; Kadhum, M.R.; Dong, Y.X.; Giddings, R.P.; Jiang, N.; Qiu, K.; Tang, J.M. Improved Performance Robustness of DSP-Enabled Flexible ROADMs Free from Optical Filters and O-E-O Conversions. J. Opt. Commun. Netw. 2016, 8, 521. [CrossRef]

25. Olmedo, M.I.; Zuo, T.; Jensen, J.B.; Zhong, Q.; Xu, X.; Popov, S.; Monroy, I.T. Multiband Carrierless Amplitude Phase Modulation for High Capacity Optical Data Links. J. Lightwave Technol. 2014, 32, 798-804. [CrossRef]

26. Shannon, C.E. Communication in the Presence of Noise. Proc. IEEE 1949, 72, 1192-1201. [CrossRef] 